\title{
Carotid Artery Disease and Stroke: Assessing Risk with Vessel Wall MRI
}

\author{
William S. Kerwin ${ }^{1,2}$ \\ ${ }^{1}$ Department of Radiology, University of Washington, Seattle, WA 98109, USA \\ ${ }^{2}$ VPDiagnostics Incorporation, Seattle, WA 98101, USA \\ Correspondence should be addressed to William S. Kerwin, bkerwin@u.washington.edu
}

Received 26 August 2012; Accepted 3 October 2012

Academic Editors: A. Becker, F. Quaini, and J. J. van Lieshout

Copyright ( 2012 William S. Kerwin. This is an open access article distributed under the Creative Commons Attribution License, which permits unrestricted use, distribution, and reproduction in any medium, provided the original work is properly cited.

\begin{abstract}
Although MRI is widely used to diagnose stenotic carotid arteries, it also detects characteristics of the atherosclerotic plaque itself, including its size, composition, and activity. These features are emerging as additional risk factors for stroke that can be feasibly acquired clinically. This paper summarizes the state of evidence for a clinical role for MRI of carotid atherosclerosis.
\end{abstract}

\section{Introduction}

Atherosclerotic disease is the leading cause of death and disability in the United States and worldwide [1,2]. Outside of the coronary circulation, the carotid arteries are likely the most clinically significant site of atherosclerosis. Estimates place carotid atherosclerosis as the cause of as many as $20 \%$ of all ischemic strokes [3]. This association led to 1.35 million carotid endarterectomy (CEA) procedures between 1998 and 2008 in the United States in patients deemed at high risk for stroke, in addition to 90,000 carotid stenting (CAS) procedures [4].

At present, the risk of stroke, on which clinical indications for CEA or CAS are based, is determined primarily from the percentage of stenosis of the vessel due to blockage of the lumen by the atherosclerotic plaque. Large trials of CEA including the North American Symptomatic Carotid Endarterectomy Trial have shown a $17 \%$ reduction in absolute risk of stroke over two years in patients with recent cerebrovascular symptoms and high-grade carotid stenosis [5]. In asymptomatic patients, studies such as the Asymptomatic Carotid Atherosclerosis Study have shown more modest benefits of CEA in patients with high-grade stenosis [6]. Advances in treatment since completion of these studies, including widespread use of statins, have further eroded the perceived benefit of intervention in asymptomatic patients leading many to advocate only medical therapy in the absence of cerebrovascular symptoms $[7,8]$. On the other hand, studies have shown that in high-risk patients without any measurable stenosis, advanced plaques are present in a high proportion [9]. Thus, basing decisions on stenosis alone leads to overtreatment of select populations, whereas other populations with lower degrees of stenosis may be undertreated. These controversies and the potential to better manage both symptomatic and asymptomatic patients have fueled the efforts of numerous researchers to identify additional markers of stroke risk that better identify patients who will benefit from intervention.

Magnetic resonance imaging (MRI) is emerging as the best candidate for augmenting stenosis with additional diagnostic features pertinent to patient management. Numerous MRI studies have identified features of carotid plaque associated with both prior and subsequent cerebrovascular events. Ongoing studies such as our own SmartRisk trial (ClinicalTrials.gov identifier no. NCT00860184) are prospectively testing the ability of carotid MRI to predict stroke in a clinical environment. In this paper, the current state of the art and future directions for assessing risk of stroke by carotid MRI are presented.

\section{Intraplaque Hemorrhage in Carotid Atherosclerosis}

To date, the feature that has garnered the most attention for its association with stroke has been intraplaque hemorrhage (IPH). Prospective studies of patients with IPH 
have yielded a consistent pattern of higher stroke rates for patients with IPH (Table 1) [10-15]. The etiology of IPH is somewhat unclear. The bulk of IPH is thought to arise from immature and leaky neovessels that have vascularized established plaques [16]. The membranes of red blood cells in IPH may be a predominant source of free cholesterol within the necrotic cores of plaques [17]. IPH is also a potent proinflammatory stimulus, leading to accumulations of macrophages that release proteinases, thereby, degrading the fibrous cap that separates the thrombogenic core of the plaque from the lumen $[17,18]$. Rupture of the fibrous cap with subsequent thrombosis is the most common feature of carotid plaques implicated in stroke and is more common in those with larger necrotic core size, cap inflammation, and IPH [19].

MRI of IPH is facilitated by the presence of methemoglobin in IPH, which serves as an endogenous contrast agent leading to shortened longitudinal relaxation constant T1 and hyperintensity on T1-weighted images [20, 21]. Hyperintensity on T1-weighted images has high sensitivity and specificity for presence of IPH (Table 2) [20-26]. The comparative simplicity of IPH detection with MRI, its good diagnostic accuracy, and the clinical importance of IPH, make MRI detection of IPH the most likely near-term addition to carotid artery diagnostics in the clinic.

In terms of imaging approaches, several options are available. Our group reported an association of IPH with high signals on both spin echo and time-of-flight (TOF) gradient echo T1-weighted images more than a decade ago (Figure 1) [27]. In parallel, techniques for direct imaging of deep vein thrombosis were being developed based on an inversion recovery (IR) magnetization prepared rapid gradient echo (MP-RAGE) sequence [28]. Subsequent application of this technique to carotid atherosclerosis demonstrated its ability to detect IPH as well [20]. In a head-to-head comparison of accuracy for detection of IPH, Ota et al. found the MP-RAGE sequence outperformed both TOF and spin echo approaches [25]. The key feature of the MP-RAGE sequence is the IR preparation which yields both strong T1 weighting in the plaque and suppression of inflowing blood in the vessel lumen for high contrast. In fact, addition of phase-sensitive reconstruction can further increase this contrast [29].

A recent development has been the combination of IPH imaging with MR angiography (MRA) for highly efficient imaging of both stenosis and IPH. One approach evaluates maximum intensity projection (MIP) views of TOF MRA results for the presence of juxtaluminal hyperintensities [24]. The mask image from contrast-enhanced MRA acquisitions can also be used to detect IPH and has led to greater detection accuracy than TOF [26]. A highly promising technique is the simultaneous noncontrast angiography and IPH (SNAP) imaging approach, which uses phase-sensitive reconstruction of an IR-prepared sequence that is timed to produce strongly negative signals in the vessel lumen and strongly positive signals in IPH [30]. This leads to natural separation of MRA (negative) and IPH (positive) signals, with overall high contrast with background tissues (Figure 2).

Evidence for the clinical significance of MRI-detected carotid IPH is widespread, although the studies tend to be small in number of participants. In the largest prospective study to date, 154 asymptomatic subjects with $50-79 \%$ stenosis were imaged at baseline and followed for an average of 38.2 months [10]. Of these, the $28 \%$ of subjects with IPH accounted for $67 \%$ of cerebrovascular events. In a similar study of 91 initially asymptomatic men with $50-70 \%$ stenosis followed for a mean period of 25 months, all of the six cerebrovascular events occurred in arteries with IPH present at baseline [13]. This population is especially important because trials of CEA have failed to show a benefit of surgery for asymptomatic patients with moderate stenosis, despite a 5 -year stroke risk of 9.5\% [31].

Another critical population is symptomatic patients with moderate stenosis who also do not benefit from CEA, but may have a 5 -year stroke risk topping $18 \%$ [32]. In a study of 64 symptomatic patients with $30-69 \%$ stenosis followed for a mean period of 28 months, $39(61 \%)$ demonstrated IPH on MRI and accounted for 13 out of 14 ipsilateral ischemic events [12]. This study is reinforced by a report on 66 symptomatic patients with high-grade stenosis awaiting CEA, wherein $88 \%$ of the 17 recurrent events occurred in the 44 subjects $(67 \%)$ with IPH [11]. A similar study of 62 symptomatic subjects with $70-99 \%$ stenosis also reported a higher rate of recurrent events in those with IPH and found similarly high rates of recurrence in both moderate (30-69\%) and high-grade (70-99\%) stenosis when IPH was present [14]. Finally, a further study of 61 symptomatic patients with a range of stenoses indicated an elevated risk for recurrent events when IPH was present [15].

In addition to these key prospective studies linking IPH to future stroke and transient ischemic attack, a number of other studies lend credence to the relevance of IPH in carotid atherosclerosis. Several studies have reported that IPH is more common in patients with a recent history of ischemic symptoms compared to asymptomatic subjects with similar stenosis [26, 33-36]. In patients with unilateral symptoms, $\mathrm{IPH}$ is more common in the ipsilateral artery than the contralateral artery and is associated with the presence of ischemic brain lesions [37-39]. IPH is also associated with more rapid growth of the carotid lesion and with the development of surface irregularities consistent with fibrous cap rupture [40-42].

To transition into clinical practice, MRI detection of IPH still needs to undergo trials comparing outcomes among different treatment options for patients with and without IPH. Toward this end, preliminary studies have been performed comparing CEA and CAS. In one study, the existence of hyperintense signal on T1-weighted images of carotid lesions was investigated in 56 patients undergoing CAS versus 25 patients undergoing CEA [43]. Among patients positive for T1-weighted hyperintensity, those receiving CAS were significantly more likely to develop silent ischemic brain lesions than those receiving CEA (61\% versus 13\%; $P=$ 0.006). Among another 112 patients undergoing CAS, the presence of hyperintense signals consistent with IPH on TOF images was found to yield significantly higher likelihood of periprocedural symptoms (18.4\% versus $1.4 \% ; P=0.003$ ) [44]. Thus, IPH identified by MRI may be helpful for determining the appropriateness of CAS. 
TABLE 1: Summary of studies reporting risk of cerebrovascular ischemic events comparing patients with and without intraplaque hemorrhage.

\begin{tabular}{lccccc}
\hline Source & $N$ & Symptomatic? & Stenosis & Hazard ratio & $P$ \\
\hline Takaya et al. 2006 [10] & 154 & No & $50-79 \%$ & 5.2 & 0.005 \\
Altaf et al. 2007 [11] & 66 & Yes & $60-99 \%$ & 4.8 & $<0.05$ \\
Altaf et al. 2008 [12] & 64 & Yes & $30-69 \%$ & 9.8 & 0.03 \\
Singh et al. 2009 [13] & 98 & No & $50-70 \%$ & 3.59 & $<0.001$ \\
Kurosaki et al. 2011 [14] & 62 & Yes & $70-99 \%$ & NR & 0.051 \\
Sadat et al. 2010 [15] & 61 & Yes & NR & 5.85 & 0.02 \\
\hline
\end{tabular}

NR: Not reported.

TABLE 2: Summary of studies reporting sensitivity and specificity for MRI detection of intraplaque hemorrhage in carotid atherosclerosis.

\begin{tabular}{lccc}
\hline Source & Imaging method(s) & Sensitivity (\%) & Specificity (\%) \\
\hline Moody et al. 2003 [20] & MP-RAGE & 84 & 84 \\
Chu et al. 2004 [21] & FSE and TOF & 90 & 74 \\
Kampschulte et al. 2004 [22] & FSE and TOF & 96 & 82 \\
Bitar et al. 2008 [23] & GRE & 100 & 80 \\
Yim et al. 2008 [24] & TOF & 81 & 83 \\
Ota et al. 2010 [25] & MP-RAGE & 87 & 97 \\
Qiao et al. 2011 [26] & CE-MRA mask & 87 & 99 \\
\hline
\end{tabular}

\section{Plaque Burden}

Considerable focus has also been placed on the relevance of plaque size in determining the risk of clinical events. Plaque size is often referred to as atherosclerotic "burden," which may refer to volume, cross-sectional area, thickness, or a ratio of measurements. The interest in burden measurements arises from the observations of Glagov of compensatory enlargement of the vessel in response to plaque accumulation in the coronary arteries [45]. In arteries undergoing such positive remodeling, high-risk plaque may develop with little or no stenosis. Indeed, studies have found little association between stenosis and plaque burden $[46,47]$.

For evaluating the association of burden with risk of events, care must be taken in choosing the burden metric. Total wall volume has been shown to be highly reproducible [48-53]. However, volume is highly sensitive to the total longitudinal coverage of the artery and native artery size, making comparisons of volumes across individuals and studies difficult. Among possible burden measurements, two measurements that are least influenced by coverage and artery size are maximal wall thickness and maximal ratio of vessel wall area to total vessel area. The latter metric, called percent atheroma volume or normalized wall index (NWI), has a value of 0.4 or less in normal arteries and rises to 1.0 for an occluded artery [54].

Imaging methods for measuring plaque burden revolve around black-blood sequences, often using double inversion recovery blood suppression [55]. Regardless of the contrast weightings used, black-blood measurements of plaque burden have been found to be highly consistent and have strong correlations with ex vivo measurements [46, 56]. Two important concerns that arise, however, are flow artifacts, and partial volume effects due to obliquity of the vessel wall. When using inflow-dependent blood suppression techniques, flow artifacts result where slow or recirculating flow maintains blood within the imaging slab [57]. The resulting artifact can be difficult to distinguish from plaque (Figure 3). Obliquity between the vessel wall and imaging plane leads to apparent elongation of the vessel that can induce overestimates of wall thickening as high as $50 \%$ to $100 \%$ for some imaging geometries [58].

Both of these challenges are being addressed by new imaging methods for inflow-independent blood suppression and $3 \mathrm{D}$ imaging to minimize partial volume effects. The use of 3D sequences also leads to improved signal-to-noise ratios, increased longitudinal coverage, improved resolution especially in the through-plane direction, and greater acquisition efficiency. Balu et al. [59] utilize motion-sensitized driven equilibrium flow suppression with a fast low-angle shot readout to obtain black-blood 3D carotid artery images with $0.7 \mathrm{~mm}$ isotropic resolution. A similar black-blood preparation has also been combined with a steady-state free precession readout [60]. The sampling perfection with application optimized contrast using different flip angle evolution (SPACE) technique permits 3D spin echo imaging with native blood suppression [61].

Measuring plaque burden is enabled by programs for identifying the inner and outer boundaries of the vessel wall on cross-sectional MR images. A number of methods for automated and semiautomated boundary detection have been proposed for this purpose [53, 62-66]. In addition to the obvious time advantage of automated systems, they also have been shown to provide improvements in measurement reproducibility of plaque burden $[53,66]$.

Studies linking plaque burden to risk of cerebrovascular events are less common than those linking IPH to risk. In the same study that showed elevated hazard ratios for IPH in 


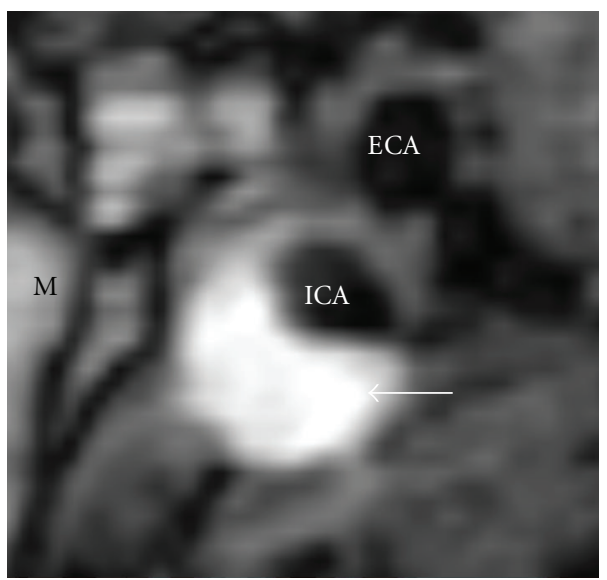

(a)

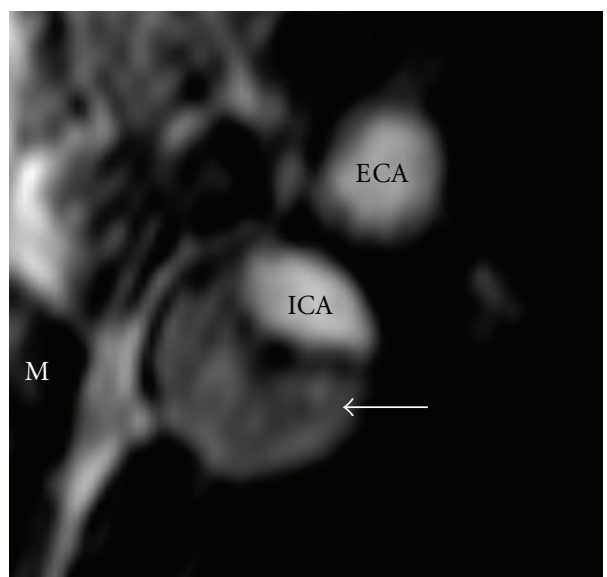

(b)

Figure 1: Appearance of intraplaque hemorrhage (arrow) on (a) T1-weighted fast spin-echo and (b) time-of-flight magnetic resonance images.

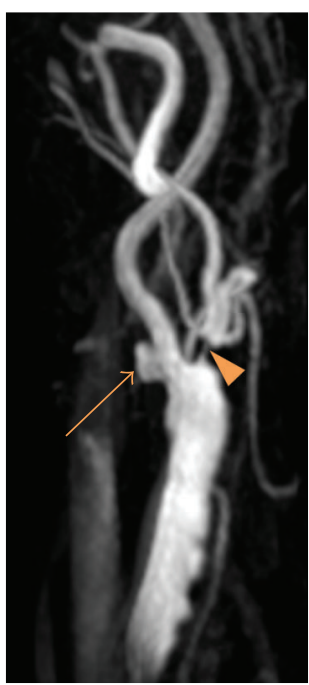

(a)

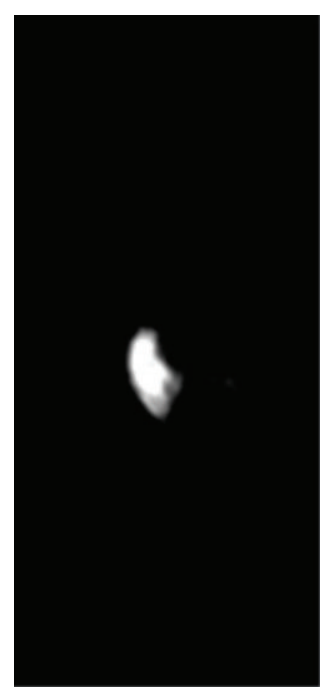

(b)

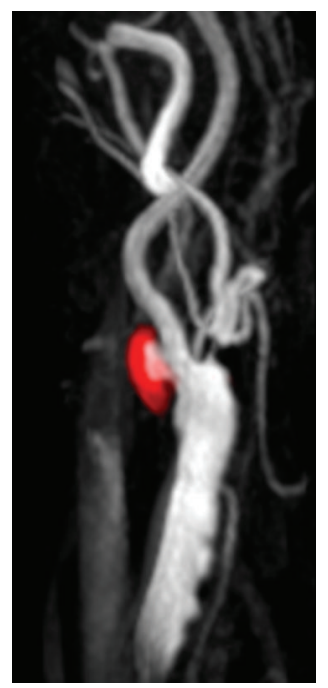

(c)

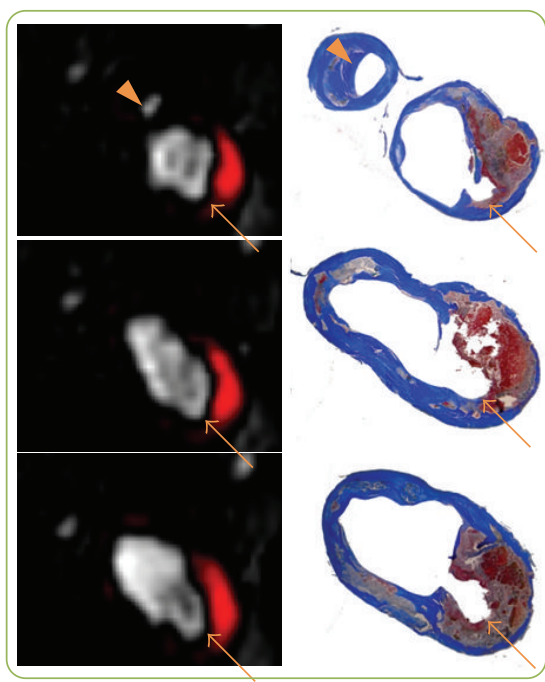

(d)

FIGURE 2: Example of simultaneous noncontrast angiography and intraplaque hemorrhage (SNAP) imaging results showing (a) the negative MRA image, (b) the positive IPH image, and (c) a fused image with the IPH in red. Cross-sectional images show good agreement with corresponding histology (d) including the presence of an ulceration (arrow) and highly stenotic external carotid artery (arrowhead). Reproduced with permission from [30].

154 asymptomatic subjects with $50-79 \%$ stenosis, maximal wall thickness was also a risk factor for subsequent stroke or TIA, with a hazard ratio of 1.6 for each $1 \mathrm{~mm}$ increase in thickness [10]. In a study combining prior stroke with other major adverse cardiac events, carotid wall thickness and area were both significantly higher in patients with prior events [67]. On the other hand, a number of studies comparing plaque burden in highly stenotic arteries with and without cerebrovascular symptoms have failed to find a significant difference in wall areas $[33,37]$. Thus, the importance of plaque burden for characterizing risk remains uncertain, although burden remains important for studies evaluating plaque progression and response to treatment [68-71].

\section{Plaque Composition}

Ultimately, complications from atherosclerosis arise as a result of biomechanical disruptions at the interface of the plaque with the vessel lumen. A further strategy for risk assessment is thus, to image the complete compositional structure of the atherosclerotic plaque (Figure 4). The most common type of disruption is rupture of the fibrous cap overlying the necrotic core [19]. Thin fibrous caps and large necrotic cores both contribute to structural weakness of the plaque. In symptomatic patients, a minimum cap thickness under 200 microns was associated with an odds ratio of 5.0 for the presence of fibrous cap rupture [72]. Larger necrotic core size and closer proximity of the necrotic core 


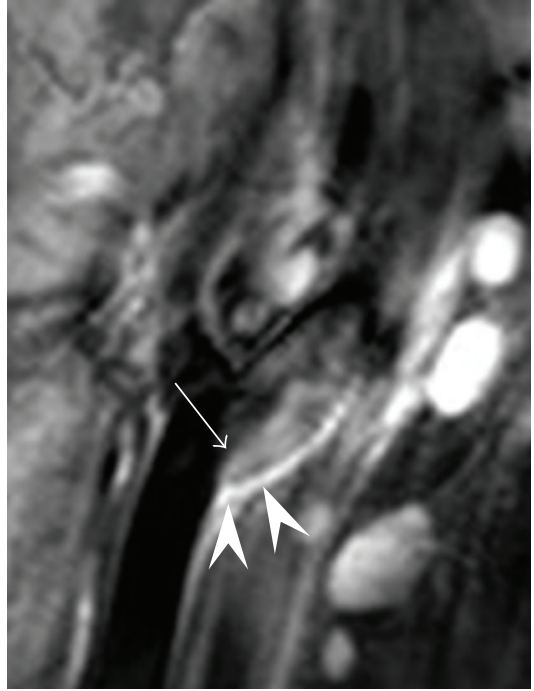

(a)

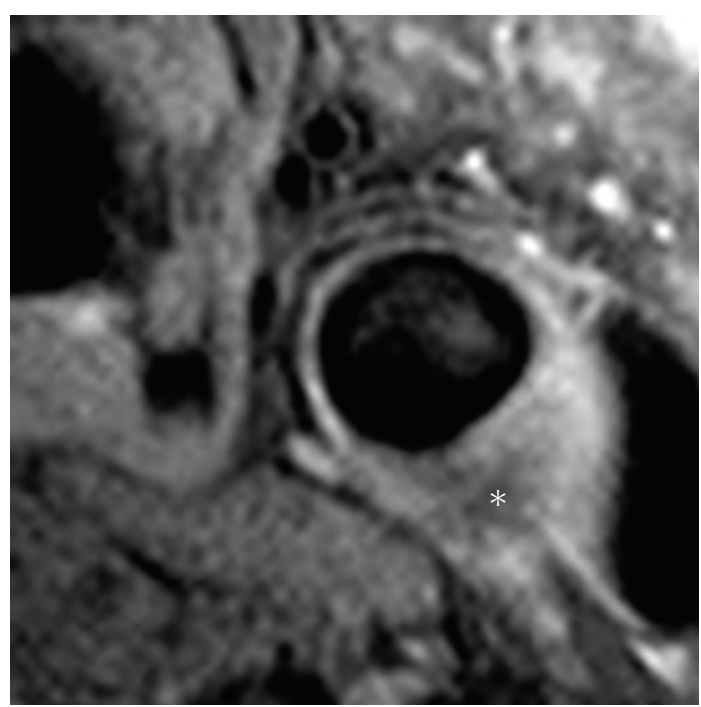

(b)

FIGURE 3: Example of flow artifact on a double inversion recovery black-blood image, caused by circulating flow in the vicinity of the carotid bulb. Longitudinal view (a) of the artifact (arrow) shows it extending from the proximal end of the bulb (arrow heads). Axial view shows the resulting plaque-mimicking artifact $(*)$.

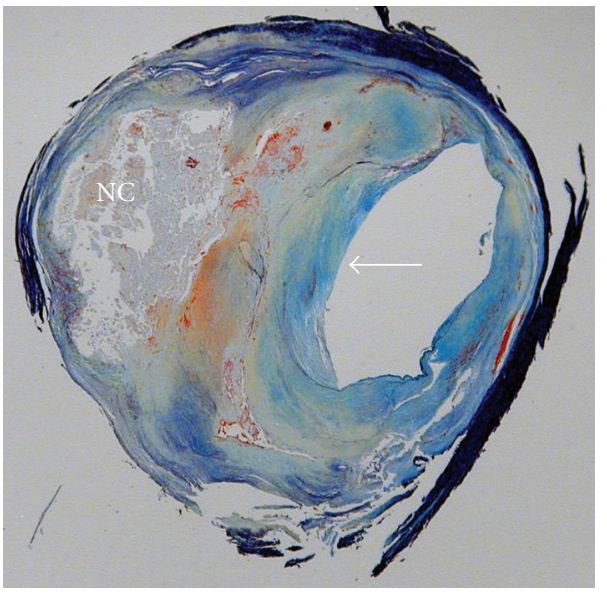

FIGURE 4: Histological specimen of advanced atherosclerotic plaque removed via carotid endarterectomy and stained with Movat's pentachrome. The lipid-rich necrotic core is labeled "NC" and the fibrous cap is indicated by an arrow.

to the lumen have also been implicated in cap rupture $[4,8]$. Plaque calcification can also contribute to risk of disruption. Specifically, calcified nodules protruding into the lumen can lead to thrombus [73]. Calcified nodules are distinguished from most plaque calcifications, which are separated from the lumen by fibrous tissue and may be indicative of stable lesions [74].

MRI strategies for assessing structural integrity of plaque have thus focused on identifying the fibrous cap, necrotic core, and calcifications. The necrotic core may also be divided into lipid-rich core and core with IPH. The current approach revolves around a multicontrast protocol of $2 \mathrm{D}$
TABle 3: Criteria for determining plaque components from common MRI contrast weightings (adapted from $[75,76]$ ).

\begin{tabular}{lcccc}
\hline \multirow{2}{*}{ Component } & \multicolumn{4}{c}{ Contrast weighting } \\
& T1 & T2 & TOF & CET1 \\
\hline Necrotic core with IPH & + & $-/+$ & + & O \\
Necrotic core without IPH & $\mathrm{O} /+$ & - & O & - \\
Calcification & - & - & - & - \\
Dense fibrous & $\mathrm{O}$ & $\mathrm{O}$ & - & $\mathrm{O}$ \\
Loose matrix & $\mathrm{O}$ & + & $\mathrm{O}$ & + \\
\hline
\end{tabular}

Indicates signal intensity relative to adjacent sternocleidomastoid muscle: + : brighter; O: isointense; -: darker.

fast spin-echo black-blood imaging with a minimum of T1 and $\mathrm{T} 2$ contrast weightings combined with 3D TOF brightblood imaging (Figure 5). Using these three weightings and an additional proton-density-weighted scan, Saam et al. provided guidelines for identifying calcification and necrotic core among other features based on relative signal intensities in each of the contrast weightings [75]. In 214 matched crosssectional locations, the measured areas of these components correlated with corresponding histological measurements with coefficients in excess of 0.7 . Further addition of contrast-enhanced T1-weighted images using gadolinium agents improves identification of the necrotic core as a nonenhancing region [76]. Adding contrast-enhanced T1weighted images to the protocol was found to reduce the interrater coefficient of variation for measuring LRNC size from $33.5 \%$ to $17.6 \%$ [77]. Intensity characteristics of plaque components on common contrast weightings are summarized in Table 3.

Contrast-enhanced MRI is also the preferred method for identifying and measuring the fibrous cap [76]. Gadolinium 


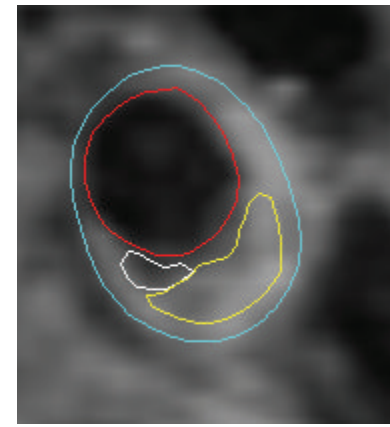

(a)

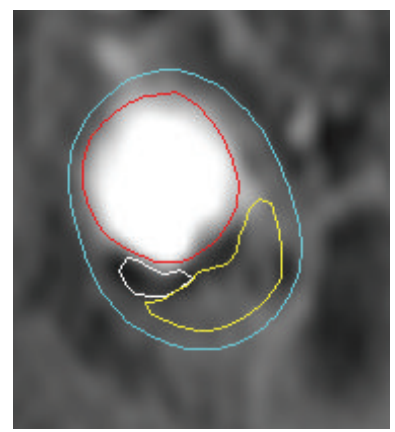

(c)

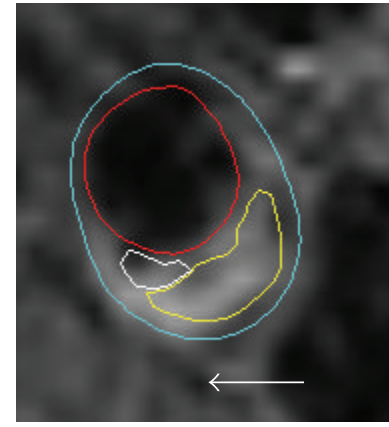

(b)

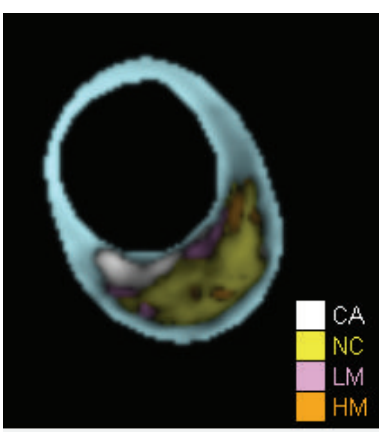

(d)

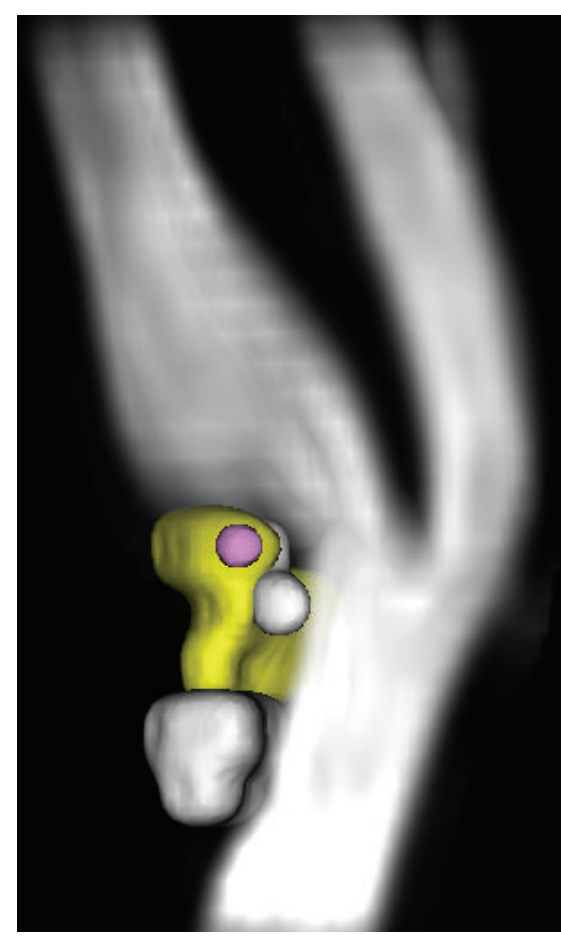

(e)

FIGURE 5: Example of multicontrast MRI characterization of carotid plaque composition showing (a) T1-weighted, (b) T2-weighted, and (c) time-of-flight images of the carotid artery. Automated segmentation with MEPPS [78] yields (d) a color-coded map indicating locations of calcification (CA), necrotic core (NC), loose matrix (LM), and hemorrhage (HM). Combination of results from cross-sections spanning the length of the plaque produce (e) a 3D rendering of the plaque components relative to the angiographic view of the carotid bifurcation (rendered with MRI-PlaqueView, VPDiagnostics Inc., Seattle, WA).

agents have been found to preferentially enhance fibrous regions $[79,80]$. This phenomenon allows MRI to make measurements of the fibrous cap that correlate with histology in CEA patients [73].

Identification of plaque components can be facilitated by automated segmentation algorithms $[64,65,78,81-85]$. Our morphology-enhanced probabilistic plaque segmentation (MEPPS) algorithm classifies plaque regions using a combination of intensity information and local plaque morphology (Figure 5(d)) [78]. In validation testing, MEPPS has proven to perform equivalent to human observers in accuracy compared to histological assessment and in reproducibility [66, $78,81,82]$. MEPPS has also been able to show a reduction in plaque necrotic core size under treatment with rosuvastatin [69].

Once the composition of the plaque is known, the complete 3D structure of the plaque can be reconstructed (Figure 5(e)). Alternatively, summary statistics can be extracted in the form of total volumes of each component, maximal areas, or percent of total plaque burden. Building upon the idea of plaque composition leading to lack of structural integrity, numerous efforts have also been made to estimate stress distributions within the plaques, based on MR images [86-88].

Utilizing these tools, numerous studies have examined the roles of the fibrous cap, necrotic core, and calcification in determining the risk of cerebrovascular events. In the comprehensive study of Takaya et al. [10], the presence of a thin or ruptured fibrous cap had the strongest association with subsequent development of symptoms, with a corresponding hazard ratio of 17 . This study also reported a significant association of necrotic core size (both absolute and as a percentage of plaque area) with development of symptoms. An association of fibrous cap rupture with subsequent events has also been reported in a symptomatic population [15]. Association of thin or ruptured caps with prior symptoms has been noted in several additional studies $[33-35,89]$. Sadat et al. found MRI measurements of actual cap thickness were larger in asymptomatic subjects than recently symptomatic ones ( 800 microns versus 600 microns) [34]. The necrotic core has also been consistently found to be more prevalent and larger in arteries implicated in a history of symptoms $[33,35,90]$. Finally, maximal stress computed based on the MRI findings has been found to be higher in plaques implicated in both prior and subsequent symptoms $[15,91]$. Regarding calcification, no significant association with symptoms has been reported [10,37].

\section{Plaque Activity}

On top of the plaque structural components, various biological processes may be superimposed. Most notably, 
inflammatory cell infiltration plays a role in cholesterol transport and fibrous cap degradation through release of matrix metalloproteinases (MMPs) $[92,93]$. Accumulations of macrophages, particularly within the fibrous cap have been consistently found to be associated with symptomatic carotid disease $[94,95]$. In conjunction with inflammation, networks of neovessels supply the metabolic needs of the plaque activity. Increased plaque neovascularization has been shown to distinguish symptomatic and asymptomatic plaques $[96,97]$.

The availability of specific cellular and molecular targets makes carotid plaque activity an attractive application for molecular imaging. Numerous efforts are underway to develop contrast agents that target specific receptors involved in plaque activity. For example, an MMP inhibitor has been coupled with a gadolinium chelate to target MMPs in atherosclerotic mice [98]. Direct targeting of macrophages has been achieved by loading nanoparticles with gadolinium and targeting the scavenger receptor-B [99]. Active neovascularization has been detected by targeting alpha(v)-beta3 integrin using nanoparticles loaded with gadolinium [100]. At present, however, these techniques are experimental and have only been used in animal models of atherosclerosis.

An alternative technique that is viable for use in humans is to inject patients with ultrasmall superparamagnetic particles of iron oxides (USPIOs), which accumulate in macrophages over a period of 24-36 hours, yielding loss of signal on subsequent images [101, 102]. The loss of signal is due to the effect of USPIOs on the MRI parameter T2*, which can be measured to provide a quantitative assessment of USPIO uptake [103]. In clinical investigations, USPIO uptake has shown independence from degree of stenosis and is greater in symptomatic patients than asymptomatic [104, 105]. Interestingly, this higher USPIO uptake occurs both ipsilateral and contralateral to the side of ischemic symptoms, suggesting a systemic component to plaque inflammation [106].

Another approach to imaging plaque activity is dynamic contrast-enhanced (DCE) imaging after administration of gadolinium agents, which can be used to assess plaque perfusion. Kinetic modeling of DCE-MRI has been shown to yield parameters that reflect neovasculature extent and permeability, both of which correlate with plaque neovessel and macrophage content $[107,108]$. The method of vasa vasorum imaging (Figure 6) portrays the results from DCEMRI as a color-coded map and has enabled differential perfusion parameters to be associated with specific plaque features $[109,110]$. Differences in perfusion have also been associated with clinical markers of cardiovascular risk, such as serum levels of C-reactive protein and high-density lipoprotein $[108,109]$. In a qualitative study of enhancement patterns, greater enhancement was found in patients with prior symptoms compared to asymptomatic controls [36].

\section{Comprehensive Risk Scores}

The end goal of carotid plaque MRI is to provide a simple indication of patient risk to inform clinical decisions. The
TABLE 4: MRI classification criteria for lesion types.

\begin{tabular}{ll}
\hline Lesion type & MRI criteria \\
\hline I/II & Normal wall appearance without thickening \\
III & Eccentric thickening without core or calcification \\
IV/V & Lesion with necrotic core \\
VI & $\begin{array}{l}\text { Complicated lesion with IPH, fibrous cap rupture, } \\
\text { and/or surface thrombus }\end{array}$ \\
VII & Calcified lesion \\
VIII & Fibrous lesion \\
\hline
\end{tabular}

large number of potentially valuable imaging markers-from burden to composition to activity-has led some to suggest that a comprehensive score based on all relevant factors is desirable. The result would be an imaging-based equivalent of the popular Framingham risk scores [111].

One approach for comprehensive lesion classification has grown out of the American Heart Association lesion type classifications system for histology [112]. Cai et al. showed that a modified version of this system could be reliably used to classify lesions based on MRI [113]. The modified lesion type definitions are summarized in Table 4 .

Of the lesion types, type VI has consistently proven to be of greatest clinical interest. The type VI lesion is a complex plaque with IPH and/or surface defect such as fibrous cap rupture, ulceration, or surface thrombus. Studies have shown that prior symptoms are associated with a higher likelihood of type VI lesions compared to asymptomatic patients $[33,114]$. In symptomatic patients, type VI lesions are more common on the side of symptoms than on the contralateral side $[37,115]$. Finally, in patients evaluated for ischemic symptoms, those with findings of brain lesions are more likely to have type VI lesions [116].

Another scoring approach has been to sum the presence or absence of IPH, ulceration/fibrous cap rupture, and lipidrich necrotic core [117]. The resulting score, called "HULC" ranges from 0 to 3 . In testing, a HULC score of 2 or greater was found to have high sensitivity and specificity for identifying prior symptoms. A high concordance between HULC $>2$ and the presence of a type VI lesion is expected.

Finally, a score based on continuous measurements of burden and composition was developed to identify lesions with IPH or surface disruption. The resulting carotid atherosclerosis score was optimized using imaging data from 344 subjects [118]. Lesions with maximal thickness less than $2 \mathrm{~mm}$ were given a score of 1 . Those thicker than $2 \mathrm{~mm}$ were scored according to maximal percentage necrotic core $(\% \mathrm{NC})$ as $2(\% \mathrm{NC}<20 \%), 3(20 \%<\% \mathrm{NC}<40 \%)$, or $4(\% \mathrm{NC}>40 \%)$. This classification system showed an area under the curve for detecting IPH of 0.91 and for detecting fibrous cap rupture of 0.93 . This system is unique in that the score was generated by selection of the statistically optimal parameters from a list of burden and compositional measurements. 

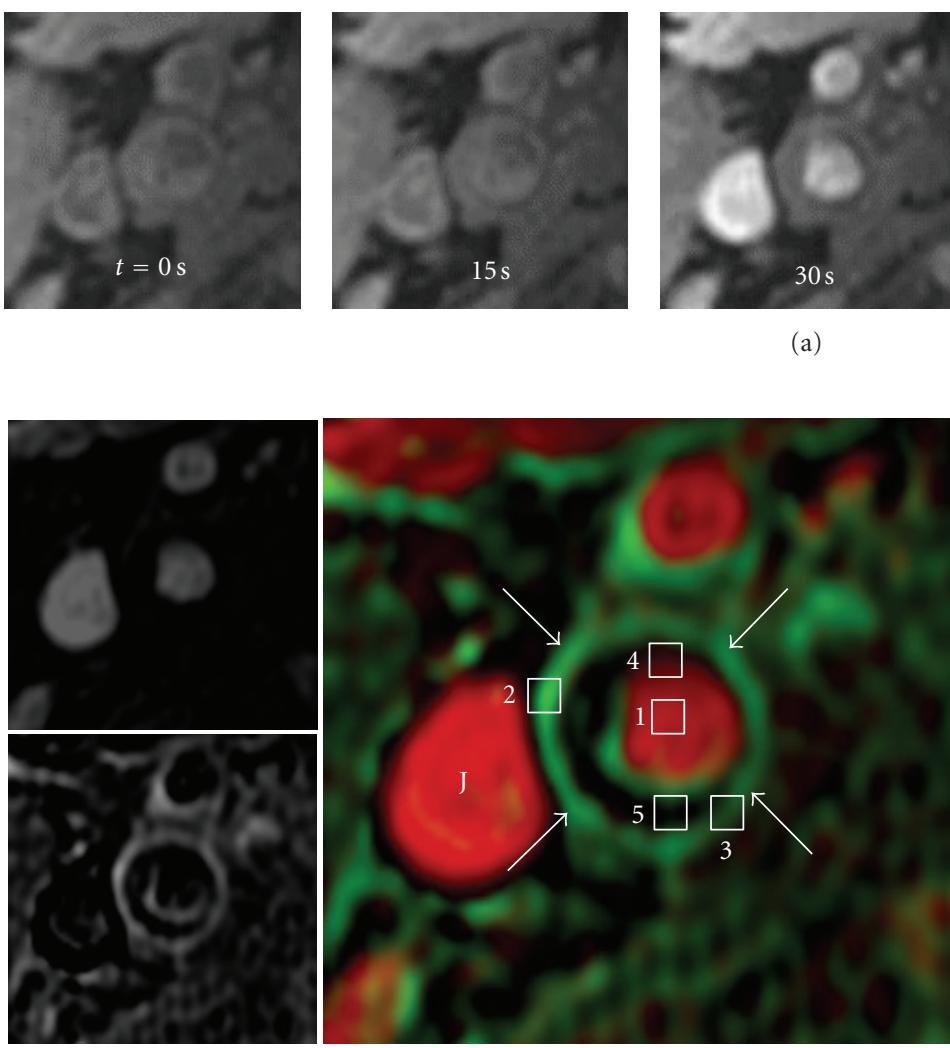

(b)

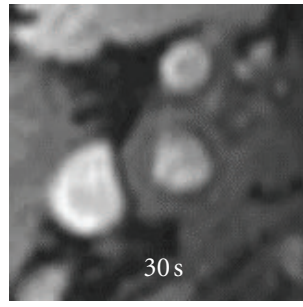

(a)
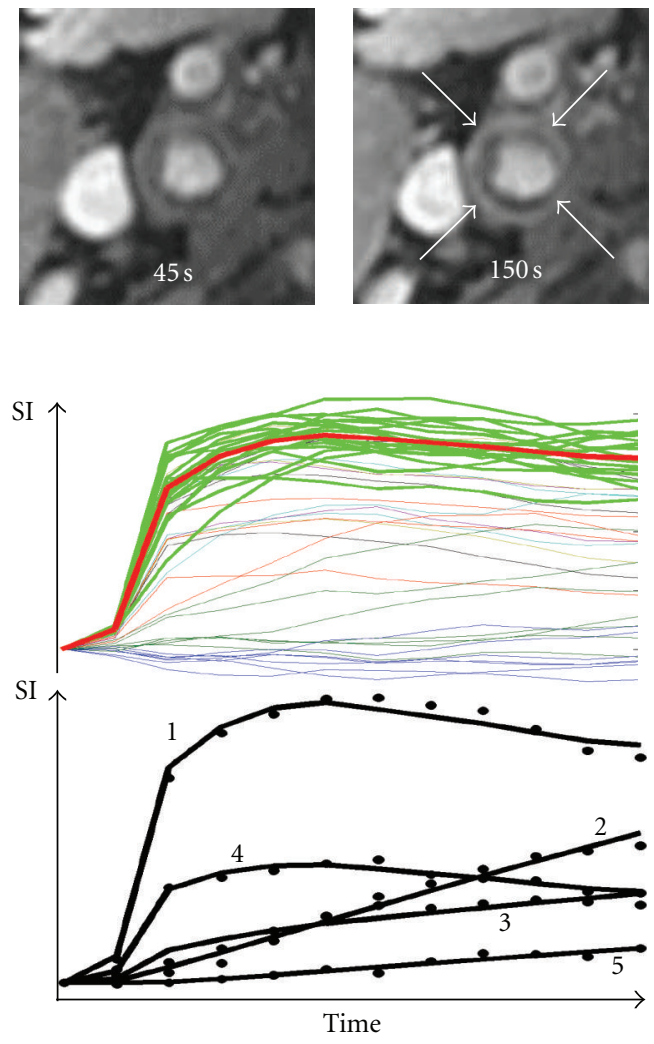

(c)

FIGURE 6: Illustration of vasa vasorum imaging via dynamic contrast-enhanced (DCE) MRI. Time series of images after injection of gadolinium contrast agents (a) are used in a kinetic model to create parametric images (b) of partial plasma volume (top left) and transfer constant (bottom left). These may be fused into the color coded image at right (arrows indicate adventitia, J: jugular vein). Sample intensity versus time curves (c) shows the actual (dotted) and modeled (solid) curves for the pixels indicated in (b) and the selection of representative blood curves (green), used in the model, from candidates. Reproduced with permission from [109].

\section{Conclusion}

In this paper, MRI techniques were presented which have the potential to predict stroke risk arising from carotid atherosclerosis. The techniques were presented in order from those most ready to be translated into clinical use to those most in need of further technological development. At the outset, techniques for detecting IPH were reviewed. No major technical hurdles in terms of imaging sequences, hardware, or postprocessing stand in the way of IPH detection in the clinic. At the other end of the spectrum, targeted contrast agents may ultimately provide the most accessible information on risk but also face considerable hurdles to gain approval for clinical use. Risk scores were discussed last because they depend on successful translation of the prior techniques.

In regards to target populations, two classes of patients stand to benefit from MRI of carotid atherosclerosis. First, patients with cryptogenic stroke, in which the cause is unknown, account for as many as $40 \%$ of all strokes [119]. Evidence suggests carotid disease is a prime culprit in many of these, but markers other than stenosis are needed to verify carotid disease as the cause [115]. A second group of patients that may benefit from carotid MRI are those with high-grade asymptomatic stenosis. In the past, these patients received a net benefit from CEA, but this benefit is waning with better medical management [7]. For both of these groups, MRI of carotid atherosclerosis has the potential to identify high-risk lesions in those patients who would benefit from intervention and the optimal type of intervention.

What is lacking, however, is proof that MRI-based factors provide marginal utility above and beyond existing risk variables. Furthermore, these techniques must yield costeffective guidance to alter current clinical guidance and yield better long-term outcomes. Ultimately, multicenter clinical trials of this technology akin to the large-scale trials of CEA are warranted $[5,6]$. The most likely scenario for these trials will be to add carotid plaque MRI to existing clinically mandated MRA or brain MRI examinations in stroke patients. The small marginal cost of the additional scanning time will allow large numbers of patients to be imaged and followed for subsequent stroke risk or benefit of intervention. The challenge for the imaging community is thus to place rapid tools for plaque analysis into the hands of stroke centers. 


\section{Abbreviations}

\begin{tabular}{|c|c|}
\hline CAS: & Carotid artery stenting \\
\hline CEA: & Carotid endarterectomy \\
\hline DCE: & Dynamic contrast-enhanced \\
\hline HULC: & Hemorrhage, ulcer, lipid core \\
\hline IPH: & Intraplaque hemorrhage \\
\hline IR: & Inversion recovery \\
\hline MEPPS: & $\begin{array}{l}\text { Morphology-enhanced probabilistic plaque } \\
\text { segmentation }\end{array}$ \\
\hline MMP: & Matrix metalloproteinase \\
\hline MP-RAGE: & $\begin{array}{l}\text { Magnetization prepared rapid acquisition } \\
\text { gradient echo }\end{array}$ \\
\hline MRA: & Magnetic resonance angiography \\
\hline MRI: & Magnetic resonance imaging \\
\hline NC: & Necrotic core \\
\hline NWI: & Normalized wall index \\
\hline SNAP: & $\begin{array}{l}\text { Simultaneous noncontrast angiograph and } \\
\text { intraplaque hemorrhage imaging }\end{array}$ \\
\hline SPACE: & $\begin{array}{l}\text { Sampling perfection with application } \\
\text { optimized contrast using different flip angle } \\
\text { evolution }\end{array}$ \\
\hline TOF & Time-of-flight \\
\hline & $\begin{array}{l}\text { Ultrasmall superparamagnetic particles of } \\
\text { iron oxide. }\end{array}$ \\
\hline
\end{tabular}

\section{Acknowledgment}

Research support provided under NIH grants R44 HL070576 and R01 HL056874.

\section{References}

[1] S. L. Murphy, J. Xu, and K. D. Kochanek, "Deaths: preliminary data for 2010," National Vital Statistics Reports, vol. 60, no. 4, pp. 1-51, 2012.

[2] World Health Organization, "Cardiovascular diseases (CVDs)," Fact Sheet no. 317, 2011.

[3] R. J. Wityk, D. Lehman, M. Klag, J. Coresh, H. Ahn, and B. Litt, "Race and sex differences in the distribution of cerebral atherosclerosis," Stroke, vol. 27, no. 11, pp. 1974-1980, 1996.

[4] T. M. Dumont and A. I. Rughani, "National trends in carotid artery revascularization surgery: clinical article," Journal of Neurosurgery, vol. 116, no. 6, pp. 1251-1257, 2012.

[5] D. W. Taylor and H. J. M. Barnett, "Beneficial effect of carotid endarterectomy in symptomatic patients with high-grade carotid stenosis," The New England Journal of Medicine, vol. 325, no. 7, pp. 445-453, 1991.

[6] J. F. Toole, "Endarterectomy for asymptomatic carotid artery stenosis," JAMA, vol. 273, no. 18, pp. 1421-1428, 1995.

[7] A. L. Abbott and G. A. Donnan, "Does the "high risk" patient with asymptomatic carotid stenosis really exist?" European Journal of Vascular and Endovascular Surgery, vol. 35, no. 5, pp. 524-533, 2008.

[8] P. A. Schneider and A. R. Naylor, "Transatlantic debate. asymptomatic carotid artery stenosis-medical therapy alone versus medical therapy plus carotid endarterectomy or stenting," European Journal of Vascular and Endovascular Surgery, vol. 40, no. 2, pp. 274-281, 2010.

[9] L. Dong, H. R. Underhill, W. Yu et al., "Geometric and compositional appearance of atheroma in an angiographically normal carotid artery in patients with atherosclerosis," American Journal of Neuroradiology, vol. 31, no. 2, pp. 311316, 2010.

[10] N. Takaya, C. Yuan, B. Chu et al., "Association between carotid plaque characteristics and subsequent ischemic cerebrovascular events: a prospective assessment with MRIinitial results," Stroke, vol. 37, no. 3, pp. 818-823, 2006.

[11] N. Altaf, S. T. MacSweeney, J. Gladman, and D. P. Auer, "Carotid intraplaque hemorrhage predicts recurrent symptoms in patients with high-grade carotid stenosis," Stroke, vol. 38, no. 5, pp. 1633-1635, 2007.

[12] N. Altaf, L. Daniels, P. S. Morgan et al., "Detection of intraplaque hemorrhage by magnetic resonance imaging in symptomatic patients with mild to moderate carotid stenosis predicts recurrent neurological events," Journal of Vascular Surgery, vol. 47, no. 2, pp. 337-342, 2008.

[13] N. Singh, A. R. Moody, D. J. Gladstone et al., "Moderate carotid artery stenosis: MR imaging-depicted intraplaque hemorrhage predicts risk of cerebrovascular ischemic events in asymptomatic men," Radiology, vol. 252, no. 2, pp. 502508, 2009.

[14] Y. Kurosaki, K. Yoshida, H. Endo, M. Chin, and S. Yamagata, "Association between carotid atherosclerosis plaque with high signal intensity on T1-weighted imaging and subsequent ipsilateral ischemic events," Neurosurgery, vol. 68, no. 1, pp. 62-67, 2011.

[15] U. Sadat, Z. Teng, V. E. Young et al., "Association between biomechanical structural stresses of atherosclerotic carotid plaques and subsequent ischaemic cerebrovascular events-a longitudinal in vivo magnetic resonance imaging-based finite element Study," EJVES Extra, vol. 40, no. 4, pp. 485-491, 2010.

[16] F. D. Kolodgie, H. K. Gold, A. P. Burke et al., "Intraplaque hemorrhage and progression of coronary atheroma," The New England Journal of Medicine, vol. 349, no. 24, pp. 23162325, 2003.

[17] R. Virmani, F. D. Kolodgie, A. P. Burke et al., "Atherosclerotic plaque progression and vulnerability to rupture: angiogenesis as a source of intraplaque hemorrhage," Arteriosclerosis, Thrombosis, and Vascular Biology, vol. 25, no. 10, pp. 20542061, 2005.

[18] A. V. Finn, M. Nakano, R. Polavarapu et al., "Hemoglobin directs macrophage differentiation and prevents foam cell formation in human atherosclerotic plaques," Journal of the American College of Cardiology, vol. 59, no. 2, pp. 166-177, 2012.

[19] J. N. E. Redgrave, J. K. Lovett, P. J. Gallagher, and P. M. Rothwell, "Histological assessment of 526 symptomatic carotid plaques in relation to the nature and timing of ischemic symptoms: the Oxford plaque study," Circulation, vol. 113, no. 19, pp. 2320-2328, 2006.

[20] A. R. Moody, R. E. Murphy, P. S. Morgan et al., "Characterization of complicated carotid plaque with magnetic resonance direct thrombus imaging in patients with cerebral ischemia," Circulation, vol. 107, no. 24, pp. 3047-3052, 2003.

[21] B. Chu, A. Kampschulte, M. S. Ferguson et al., "Hemorrhage in the atherosclerotic carotid plaque: a high-resolution MRI study," Stroke, vol. 35, no. 5, pp. 1079-1084, 2004.

[22] A. Kampschulte, M. S. Ferguson, W. S. Kerwin et al., "Differentiation of intraplaque versus juxtaluminal hemorrhage/thrombus in advanced human carotid atherosclerotic lesions by in vivo magnetic resonance imaging," Circulation, vol. 110, no. 20, pp. 3239-3244, 2004. 
[23] R. Bitar, A. R. Moody, G. Leung et al., "In vivo 3D highspatial-resolution MR imaging of intraplaque hemorrhage," Radiology, vol. 249, no. 1, pp. 259-267, 2008.

[24] J. Y. Yoo, H. C. Yeon, Y. Ko et al., "High signal intensity halo around the carotid artery on maximum intensity projection images of time-of-flight MR angiography: a new sign for intraplaque hemorrhage," Journal of Magnetic Resonance Imaging, vol. 27, no. 6, pp. 1341-1346, 2008.

[25] H. Ota, V. L. Yarnykh, M. S. Ferguson et al., "Carotid intraplaque hemorrhage imaging at 3.0-T MR imaging: comparison of the diagnostic performance of three T1weighted sequences," Radiology, vol. 254, no. 2, pp. 551-563, 2010.

[26] Y. Qiao, M. Etesami, S. Malhotra et al., "Identification of intraplaque hemorrhage on MR angiography images: a comparison of contrast-enhanced mask and time-of-flight techniques," American Journal of Neuroradiology, vol. 32, no. 3, pp. 454-459, 2011.

[27] C. Yuan, L. M. Mitsumori, M. S. Ferguson et al., "In vivo accuracy of multispectral magnetic resonance imaging for identifying lipid-rich necrotic cores and intraplaque hemorrhage in advanced human carotid plaques," Circulation, vol. 104, no. 17, pp. 2051-2056, 2001.

[28] A. R. Moody, J. G. Pollock, A. R. O'Connor, and M. Bagnall, "Lower-limb deep venous thrombosis: direct MR imaging of the thrombus," Radiology, vol. 209, no. 2, pp. 349-355, 1998.

[29] J. Wang, M. S. Ferguson, N. Balu, C. Yuan, T. S. Hatsukami, and P. Bornert, "Improved carotid intraplaque hemorrhage imaging using a slab-selective phase-sensitive inversionrecovery (SPI) sequence," Magnetic Resonance in Medicine, vol. 64 , no. 5, pp. 1332-1340, 2010.

[30] J. Wang, P. Börnert, H. Zhao et al., "Simultaneous noncontrast angiography and intraPlaque hemorrhage (SNAP) imaging for carotid atherosclerotic disease evaluation," Magnetic Resonance in Medicine. In press.

[31] A. Halliday, A. Mansfield, J. Marro et al., "Prevention of disabling and fatal strokes by successful carotid endarterectomy in patients without recent neurological symptoms: randomised controlled trial," The Lancet, vol. 363, no. 9420, pp. 1491-1502, 2004.

[32] H. J. M. Barnett, D. W. Taylor, M. Eliasziw et al., "Benefit of carotid endarterectomy in patients with symptomatic moderate or severe stenosis," The New England Journal of Medicine, vol. 339, no. 20, pp. 1415-1425, 1998.

[33] J. M. U-King-Im, T. Y. Tang, A. Patterson et al., "Characterisation of carotid atheroma in symptomatic and asymptomatic patients using high resolution MRI," Journal of Neurology, Neurosurgery and Psychiatry, vol. 79, no. 8, pp. 905-912, 2008.

[34] U. Sadat, R. A. Weerakkody, D. J. Bowden et al., "Utility of high resolution MR imaging to assess carotid plaque morphology: a comparison of acute symptomatic, recently symptomatic and asymptomatic patients with carotid artery disease," Atherosclerosis, vol. 207, no. 2, pp. 434-439, 2009.

[35] J. K. DeMarco, H. Ota, H. R. Underhill et al., "MR carotid plaque imaging and contrast-enhanced MR angiography identifies lesions associated with recent ipsilateral thromboembolic symptoms: an in vivo study at 3T," American Journal of Neuroradiology, vol. 31, no. 8, pp. 1395-1402, 2010.

[36] Y. Qiao, M. Etesami, B. C. Astor, S. R. Zeiler, H. H. Trout III, and B. A. Wasserman, "Carotid plaque neovascularization and hemorrhage detected by MR imaging are associated with recent cerebrovascular ischemic events," American Journal of Neuroradiology, vol. 33, no. 4, pp. 755-760, 2012.
[37] T. Saam, J. Cai, L. Ma et al., "Comparison of symptomatic and asymptomatic atherosclerotic carotid plaque features with in vivo MR imaging," Radiology, vol. 240, no. 2, pp. 464472, 2006.

[38] R. E. Murphy, A. R. Moody, P. S. Morgan et al., "Prevalence of complicated carotid atheroma as detected by magnetic resonance direct thrombus imaging in patients with suspected carotid artery stenosis and previous acute cerebral ischemia," Circulation, vol. 107, no. 24, pp. 3053-3058, 2003.

[39] J. S. McNally, S.-E. Kim, H.-C. Yoon et al., "Carotid magnetization-prepared rapid acquisition with gradient-echo signal is associated with acute territorial cerebral ischemic events detected by diffusion-weighted MRI," Circulation, vol. 5, no. 3, pp. 376-382, 2012.

[40] H. R. Underhill, C. Yuan, V. L. Yarnykh et al., "Predictors of surface disruption with MR imaging in asymptomatic carotid artery stenosis," American Journal of Neuroradiology, vol. 31, no. 3, pp. 487-493, 2010.

[41] N. Takaya, C. Yuan, B. Chu et al., "Presence of intraplaque hemorrhage stimulates progression of carotid atherosclerotic plaques: a high-resolution magnetic resonance imaging study," Circulation, vol. 111, no. 21, pp. 2768-2775, 2005.

[42] H. R. Underhill, C. Yuan, V. L. Yarnykh et al., "Arterial remodeling in the subclinical carotid artery: a natural history study," Journal of the American College of Cardiology, vol. 2, no. 12, pp. 1381-1389, 2009.

[43] K. Yamada, S. Yoshimura, M. Kawasaki et al., "Embolic complications after carotid artery stenting or carotid endarterectomy are associated with tissue characteristics of carotid plaques evaluated by magnetic resonance imaging," Atherosclerosis, vol. 215, no. 2, pp. 399-404, 2011.

[44] S. Yoshimura, K. Yamada, M. Kawasaki et al., "High-intensity signal on time-of-flight magnetic resonance angiography indicates carotid plaques at high risk for cerebral embolism during stenting," Stroke, vol. 42, no. 11, pp. 3132-3137, 2011.

[45] S. Glagov, E. Weisenberg, and C. K. Zarins, "Compensatory enlargement of human atherosclerotic coronary arteries," The New England Journal of Medicine, vol. 316, no. 22, pp. 1371-1375, 1987.

[46] Y. Luo, N. Polissar, C. Han et al., "Accuracy and uniqueness of three in vivo measurements of atherosclerotic carotid plaque morphology with black blood MRI," Magnetic Resonance in Medicine, vol. 50, no. 1, pp. 75-82, 2003.

[47] X. Zhao, H. R. Underhill, Q. Zhao et al., "Discriminating carotid atherosclerotic lesion severity by luminal stenosis and plaque burden: a comparison utilizing high-resolution magnetic resonance imaging at 3.0 tesla," Stroke, vol. 42, no. 2, pp. 347-353, 2011.

[48] T. Saam, W. S. Kerwin, B. Chu et al., "Sample size calculation for clinical trials using magnetic resonance imaging for the quantitative assessment of carotid atherosclerosis," Journal of Cardiovascular Magnetic Resonance, vol. 7, no. 5, pp. 799808, 2005.

[49] A. Varghese, L. A. Crowe, R. H. Mohiaddin et al., "Interstudy reproducibility of three-dimensional volume-selective fast spin echo magnetic resonance for quantifying carotid artery wall volume," Journal of Magnetic Resonance Imaging, vol. 21, no. 2, pp. 187-191, 2005.

[50] M. A. Syed, J. N. Oshinski, C. Kitchen, A. Ali, R. J. Charnigo, and A. A. Quyyumi, "Variability of carotid artery measurements on 3-Tesla MRI and its impact on sample size calculation for clinical research," International Journal of Cardiovascular Imaging, vol. 25, no. 6, pp. 581-589, 2009. 
[51] F. Li, V. L. Yarnykh, T. S. Hatsukami et al., "Scan-rescan reproducibility of carotid atherosclerotic plaque morphology and tissue composition measurements using multicontrast MRI at 3T," Journal of Magnetic Resonance Imaging, vol. 31, no. 1, pp. 168-176, 2010.

[52] B. A. Wasserman, B. C. Astor, A. Richey Sharrett, C. Swingen, and D. Catellier, "MRI measurements of carotid plaque in the atherosclerosis risk in communities (ARIC) study: methods, reliability and descriptive statistics," Journal of Magnetic Resonance Imaging, vol. 31, no. 2, pp. 406-415, 2010.

[53] R. Van't Klooster, P. J. H. De Koning, R. A. Dehnavi et al., "Automatic lumen and outer wall segmentation of the carotid artery using deformable three-dimensional models in MR angiography and vessel wall images," Journal of Magnetic Resonance Imaging, vol. 35, no. 1, pp. 156-165, 2012.

[54] T. Saam, C. Yuan, B. Chu et al., "Predictors of carotid atherosclerotic plaque progression as measured by noninvasive magnetic resonance imaging," Atherosclerosis, vol. 194, no. 2, pp. e34-e42, 2007.

[55] V. L. Yarnykh and C. Yuan, "Multislice double inversionrecovery black-blood imaging with simultaneous slice reinversion," Journal of Magnetic Resonance Imaging, vol. 17, no. 4, pp. 478-483, 2003.

[56] S. Zhang, T. S. Hatsukami, N. L. Polissar, C. Han, and C. Yuan, "Comparison of carotid vessel wall area measurements using three different contrast-weighted black blood $\mathrm{mr}$ imaging techniques," Magnetic Resonance Imaging, vol. 19, no. 6, pp. 795-802, 2001.

[57] D. A. Steinman and B. K. Rutt, "On the nature and reduction of plaque-mimicking flow artifacts in black blood MRI of the carotid bifurcation," Magnetic Resonance in Medicine, vol. 39, no. 4, pp. 635-641, 1998.

[58] L. Antiga, B. A. Wasserman, and D. A. Steinman, "On the overestimation of early wall thickening at the carotid bulb by black blood MRI, with implications for coronary and vulnerable plaque imaging," Magnetic Resonance in Medicine, vol. 60 , no. 5, pp. 1020-1028, 2008.

[59] N. Balu, V. L. Yarnykh, B. Chu, J. Wang, T. Hatsukami, and C. Yuan, "Carotid plaque assessment using fast 3D isotropic resolution black-blood MRI," Magnetic Resonance in Medicine, vol. 65, no. 3, pp. 627-637, 2011.

[60] I. Koktzoglou and D. Li, "Diffusion-prepared segmented steady-state free precession: application to 3D black-blood cardiovascular magnetic resonance of the thoracic aorta and carotid artery walls," Journal of Cardiovascular Magnetic Resonance, vol. 9, no. 1, pp. 33-42, 2007.

[61] G. Mihai, Y. C. Chung, M. Kariisa, S. V. Raman, O. P. Simonetti, and S. Rajagopalan, "Initial feasibility of a multi-station high resolution three-dimensional dark blood angiography protocol for the assessment of peripheral arterial disease," Journal of Magnetic Resonance Imaging, vol. 30, no. 4, pp. 785-793, 2009.

[62] C. Yuan, E. Lin, J. Millard, and J. N. Hwang, "Closed contour edge detection of blood vessel lumen and outer wall boundaries in black-blood MR images," Magnetic Resonance Imaging, vol. 17, no. 2, pp. 257-266, 1999.

[63] H. M. Ladak, J. B. Thomas, J. R. Mitchell, B. K. Rutt, and D. A. Steinman, "A semi-automatic technique for measurement of arterial wall from black blood MRI," Medical Physics, vol. 28, no. 6, pp. 1098-1107, 2001.

[64] C. Han, W. S. Kerwin, T. S. Hatsukami, J. N. Hwang, and C. Yuan, "Detecting objects in image sequences using rulebased control in an active contour model," IEEE Transactions on Biomedical Engineering, vol. 50, no. 6, pp. 705-710, 2003.
[65] I. M. Adame, R. J. Van Der Geest, B. A. Wasserman, M. A. Mohamed, J. H. C. Reiber, and B. P. F. Lelieveldt, "Automatic segmentation and plaque characterization in atherosclerotic carotid artery MR images," Magnetic Resonance Materials in Physics, Biology and Medicine, vol. 16, no. 5, pp. 227-234, 2004.

[66] W. Kerwin, D. Xu, F. Liu et al., "Magnetic resonance imaging of carotid atherosclerosis: plaque analysis," Topics in Magnetic Resonance Imaging, vol. 18, no. 5, pp. 371-378, 2007.

[67] V. Mani, P. Muntner, S. S. Gidding et al., "Cardiovascular magnetic resonance parameters of atherosclerotic plaque burden improve discrimination of prior major adverse cardiovascular events," Journal of Cardiovascular Magnetic Resonance, vol. 11, no. 1, article 10, 2009.

[68] R. Corti, V. Fuster, Z. A. Fayad et al., "Lipid lowering by simvastatin induces regression of human atherosclerotic lesions: two years' follow-up by high-resolution noninvasive magnetic resonance imaging," Circulation, vol. 106, no. 23, pp. 2884-2887, 2002.

[69] H. R. Underhill, C. Yuan, X. Q. Zhao et al., "Effect of rosuvastatin therapy on carotid plaque morphology and composition in moderately hypercholesterolemic patients: a high-resolution magnetic resonance imaging trial," American Heart Journal, vol. 155, no. 3, pp. 584.e1-584.e8, 2008.

[70] J. M. S. Lee, M. D. Robson, L. M. Yu et al., "Effects of high-dose modified-release nicotinic acid on atherosclerosis and vascular function: a randomized, placebo-controlled, magnetic resonance imaging study," Journal of the American College of Cardiology, vol. 54, no. 19, pp. 1787-1794, 2009.

[71] X.-Q. Zhao, L. Dong, T. Hatsukami et al., "MR imaging of carotid plaque composition during lipid-lowering therapy: a prospective assessment of effect and time course," Journal of the American College of Cardiology, vol. 4, no. 9, pp. 977-986, 2011.

[72] J. N. Redgrave, P. Gallagher, J. K. Lovett, and P. M. Rothwell, "Critical cap thickness and rupture in symptomatic carotid plaques: the oxford plaque study," Stroke, vol. 39, no. 6, pp. 1722-1729, 2008.

[73] R. Virmani, E. R. Ladich, A. P. Burke, and F. D. Kolodgie, "Histopathology of carotid atherosclerotic disease," Neurosurgery, vol. 59, no. 5, pp. S219-S3, 2006.

[74] R. Virmani, F. D. Kolodgie, A. P. Burke, A. Farb, and S. M. Schwartz, "Lessons from sudden coronary death: a comprehensive morphological classification scheme for atherosclerotic lesions," Arteriosclerosis, Thrombosis, and Vascular Biology, vol. 20, no. 5, pp. 1262-1275, 2000.

[75] T. Saam, M. S. Ferguson, V. L. Yarnykh et al., "Quantitative evaluation of carotid plaque composition by in vivo MRI," Arteriosclerosis, Thrombosis, and Vascular Biology, vol. 25, no. 1, pp. 234-239, 2005.

[76] J. Cai, T. S. Hatsukami, M. S. Ferguson et al., "In vivo quantitative measurement of intact fibrous cap and lipidrich necrotic core size in atherosclerotic carotid plaque: comparison of high-resolution, contrast-enhanced magnetic resonance imaging and histology," Circulation, vol. 112, no. 22, pp. 3437-3444, 2005.

[77] N. Takaya, J. Cai, M. S. Ferguson et al., "Intra- and interreader reproducibility of magnetic resonance imaging for quantifying the lipid-rich necrotic core is improved with gadolinium contrast enhancement," Journal of Magnetic Resonance Imaging, vol. 24, no. 1, pp. 203-210, 2006. 
[78] F. Liu, D. Xu, M. S. Ferguson et al., "Automated in vivo segmentation of carotid plaque MRI with morphology-enhanced probability maps," Magnetic Resonance in Medicine, vol. 55, no. 3, pp. 659-668, 2006.

[79] C. Yuan, W. S. Kerwin, M. S. Ferguson et al., "Contrastenhanced high resolution MRI for atherosclerotic carotid artery tissue characterization," Journal of Magnetic Resonance Imaging, vol. 15, no. 1, pp. 62-67, 2002.

[80] B. A. Wasserman, W. I. Smith, H. H. Trout, R. O. Cannon, R. S. Balaban, and A. E. Arai, "Carotid artery atherosclerosis: in vivo morphologic characterization with gadoliniumenhanced double-oblique MR imaging-initial results," Radiology, vol. 223, no. 2, pp. 566-573, 2002.

[81] W. S. Kerwin, F. Liu, V. Yarnykh et al., "Signal features of the atherosclerotic plaque at 3.0 Tesla versus 1.5 Tesla: impact on automatic classification," Journal of Magnetic Resonance Imaging, vol. 28, no. 4, pp. 987-995, 2008.

[82] W. Liu, N. Balu, J. Sun et al., "Segmentation of carotid plaque using multicontrast 3D gradient echo MRI," Journal of Magnetic Resonance Imaging, vol. 35, no. 4, pp. 812-819, 2012.

[83] B. C. Te Boekhorst, R. Van 'T Klooster, S. M. Bovens et al., "Evaluation of multicontrast MRI including fat suppression and inversion recovery spin echo for identification of intraplaque hemorrhage and lipid core in human carotid plaque using the mahalanobis distance measure," Magnetic Resonance in Medicine, vol. 67, no. 6, pp. 1764-1775, 2012.

[84] C. Karmonik, P. Basto, K. Vickers et al., "Quantitative segmentation of principal carotid atherosclerotic lesion components by feature space analysis based on multicontrast MRI at 1.5 T," IEEE Transactions on Biomedical Engineering, vol. 56, no. 2, pp. 352-360, 2009.

[85] J. M. A. Hofman, W. J. Branderhorst, H. M. M. Ten Eikelder et al., "Quantification of atherosclerotic plaque components using in vivo MRI and supervised classifiers," Magnetic Resonance in Medicine, vol. 55, no. 4, pp. 790-799, 2006.

[86] D. Tang, C. Yang, J. Zheng et al., "3D MRI-based multicomponent FSI models for atherosclerotic plaques," Annals of Biomedical Engineering, vol. 32, no. 7, pp. 947-960, 2004.

[87] S. A. Kock, J. V. Nygaard, N. Eldrup et al., "Mechanical stresses in carotid plaques using MRI-based fluid-structure interaction models," Journal of Biomechanics, vol. 41, no. 8, pp. 1651-1658, 2008.

[88] H. Gao, Q. Long, M. Graves, J. H. Gillard, and Z. Y. Li, "Carotid arterial plaque stress analysis using fluid-structure interactive simulation based on in-vivo magnetic resonance images of four patients," Journal of Biomechanics, vol. 42, no. 10, pp. 1416-1423, 2009.

[89] C. Yuan, S. X. Zhang, N. L. Polissar et al., "Identification of fibrous cap rupture with magnetic resonance imaging is highly associated with recent transient ischemic attack or stroke," Circulation, vol. 105, no. 2, pp. 181-185, 2002.

[90] V. C. Cappendijk, A. G. H. Kessels, S. Heeneman et al., "Comparison of lipid-rich necrotic core size in symptomatic and asymptomatic carotid atherosclerotic plaque: initial results," Journal of Magnetic Resonance Imaging, vol. 27, no. 6, pp. 1356-1361, 2008.

[91] H. Gao, Q. Long, S. Kumar Das et al., "Study of carotid arterial plaque stress for symptomatic and asymptomatic patients," Journal of Biomechanics, vol. 44, no. 14, pp. 25512557, 2011.

[92] M. Cuchel and D. J. Rader, "Macrophage reverse cholesterol transport: key to the regression of atherosclerosis?" Circulation, vol. 113, no. 21, pp. 2548-2555, 2006.
[93] A. C. Newby, "Metalloproteinase expression in monocytes and macrophages and its relationship to atherosclerotic plaque instability," Arteriosclerosis, Thrombosis, and Vascular Biology, vol. 28, no. 12, pp. 2108-2114, 2008.

[94] S. Jander, M. Sitzer, R. Schumann et al., "Inflammation in high-grade carotid stenosis: a possible role for macrophages and T cells in plaque destabilization," Stroke, vol. 29, no. 8, pp. 1625-1630, 1998.

[95] T. Husain, C. R. Abbott, D. J. A. Scott, and M. J. Gough, "Macrophage accumulation within the cap of carotid atherosclerotic plaques is associated with the onset of cerebral ischemic events," Journal of Vascular Surgery, vol. 30, no. 2, pp. 269-276, 1999.

[96] R. Mofidi, T. B. Crotty, P. McCarthy, S. J. Sheehan, D. Mehigan, and T. V. Keaveny, "Association between plaque instability, angiogenesis and symptomatic carotid occlusive disease," British Journal of Surgery, vol. 88, no. 7, pp. 945-950, 2001.

[97] M. J. McCarthy, I. M. Loftus, M. M. Thompson et al., "Angiogenesis and the atherosclerotic carotid plaque: an association between symptomatology and plaque morphology," Journal of Vascular Surgery, vol. 30, no. 2, pp. 261-268, 1999.

[98] E. Lancelot, V. Amirbekian, I. Brigger et al., "Evaluation of matrix metalloproteinases in atherosclerosis using a novel noninvasive imaging approach," Arteriosclerosis, Thrombosis, and Vascular Biology, vol. 28, no. 3, pp. 425-432, 2008.

[99] M. J. Lipinski, J. C. Frias, V. Amirbekian et al., "Macrophagespecific lipid-based nanoparticles improve cardiac magnetic resonance detection and characterization of human atherosclerosis," Journal of the American College of Cardiology, vol. 2, no. 5, pp. 637-647, 2009.

[100] P. M. Winter, A. M. Morawski, S. D. Caruthers et al., "Molecular imaging of angiogenesis in early-stage atherosclerosis with alpha(v)beta3-integrin-targeted nanoparticles," Circulation, vol. 108, no. 18, pp. 2270-2274, 2003.

[101] M. E. Kool, V. C. Cappendijk, K. B. J. M. Cleutjens et al., "Accumulation of ultrasmall superparamagnetic particles of iron oxide in human atherosclerotic plaques can be detected by in vivo magnetic resonance imaging," Circulation, vol. 107, no. 19, pp. 2453-2458, 2003.

[102] R. A. Trivedi, J. M. U-King-Im, M. J. Graves et al., "In vivo detection of macrophages in human carotid atheroma: temporal dependence of ultrasmall superparamagnetic particles of iron oxide-enhanced MRI," Stroke, vol. 35, no. 7, pp. 16311635, 2004.

[103] A. J. Patterson, T. Y. Tang, M. J. Graves, K. H. Müller, and J. H. Gillard, "In vivo carotid plaque MRI using quantitative $T 2^{*}$ measurements with ultrasmall superparamagnetic iron oxide particles: a dose-response study to statin therapy," NMR in Biomedicine, vol. 24, no. 1, pp. 89-95, 2011.

[104] T. Y. Tang, S. P. S. Howarth, S. R. Miller et al., "Correlation of carotid atheromatous plaque inflammation using USPIOenhanced MR imaging with degree of luminal Stenosis," Stroke, vol. 39, no. 7, pp. 2144-2147, 2008.

[105] S. P. S. Howarth, T. Y. Tang, R. Trivedi et al., "Utility of USPIO-enhanced MR imaging to identify inflammation and the fibrous cap: a comparison of symptomatic and asymptomatic individuals," European Journal of Radiology, vol. 70, no. 3, pp. 555-560, 2009.

[106] T. Y. Tang, S. P. S. Howarth, S. R. Miller et al., "Comparison of the inflammatory burden of truly asymptomatic carotid atheroma with atherosclerotic plaques contralateral to symptomatic carotid stenosis: an ultra small superparamagnetic iron oxide enhanced magnetic resonance study," Journal of 
Neurology, Neurosurgery and Psychiatry, vol. 78, no. 12, pp. 1337-1343, 2007.

[107] W. Kerwin, A. Hooker, M. Spilker et al., "Quantitative magnetic resonance imaging analysis of neovasculature volume in carotid atherosclerotic plaque," Circulation, vol. 107, no. 6, pp. 851-856, 2003.

[108] W. S. Kerwin, K. D. O’Brien, M. S. Ferguson, N. Polissar, T. S. Hatsukami, and C. Yuan, "Inflammation in carotid atherosclerotic plaque: a dynamic contrast-enhanced MR imaging study," Radiology, vol. 241, no. 2, pp. 459-468, 2006.

[109] W. S. Kerwin, M. Oikawa, C. Yuan, G. P. Jarvik, and T. S. Hatsukami, "MR imaging of adventitial vasa vasorum in carotid atherosclerosis," Magnetic Resonance in Medicine, vol. 59, no. 3, pp. 507-514, 2008.

[110] H. Chen, J. Cai, X. Zhao et al., "Localized measurement of atherosclerotic plaque inflammatory burden with dynamic contrast-enhanced MRI," Magnetic Resonance in Medicine, vol. 64, no. 2, pp. 567-573, 2010.

[111] S. M. Grundy, G. J. Balady, M. H. Criqui et al., "Primary prevention of coronary heart disease: guidance from Framingham: a statement for healthcare professionals from the AHA task force on risk reduction," Circulation, vol. 97, no. 18, pp. 1876-1887, 1998.

[112] H. C. Stary, A. B. Chandler, R. E. Dinsmore et al., "A definition of advanced types of atherosclerotic lesions and a histological classification of atherosclerosis: a report from the Committee on Vascular Lesions of the council on arteriosclerosis, American heart association," Circulation, vol. 92, no. 5, pp. 1355-1374, 1995.

[113] J. M. Cai, T. S. Hatsukami, M. S. Ferguson, R. Small, N. L. Polissar, and C. Yuan, "Classification of human carotid atherosclerotic lesions with in vivo multicontrast magnetic resonance imaging," Circulation, vol. 106, no. 11, pp. 13681373, 2002.

[114] A. C. Lindsay, L. Biasiolli, J. M. S. Lee et al., "Plaque features associated with increased cerebral infarction after minor stroke and TIA: a prospective, case-control, 3-T carotid artery MR imaging study," Journal of the American College of Cardiology, vol. 5, no. 4, pp. 388-396, 2012.

[115] T. M. Freilinger, A. Schindler, C. Schmidt et al., "Prevalence of nonstenosing, complicated atherosclerotic plaques in cryptogenic stroke," Journal of the American College of Cardiology, vol. 5, no. 4, pp. 397-405, 2012.

[116] J. P. Parmar, W. J. Rogers, J. P. Mugler et al., "Magnetic resonance imaging of carotid atherosclerotic plaque in clinically suspected acute transient ischemic attack and acute ischemic stroke," Circulation, vol. 122, no. 20, pp. 2031-2038, 2010.

[117] L. Gury-Paquet, A. Millon, F. Salami et al., "Carotid plaque high-resolution MRI at $3 \mathrm{~T}$ : evaluation of a new imaging score for symptomatic plaque assessment," Magnetic Resonance Imaging, vol. 30, no. 10, pp. 1424-1431, 2012.

[118] H. R. Underhill, T. S. Hatsukami, J. Cai et al., "A noninvasive imaging approach to assess plaque severity: the carotid atherosclerosis score," American Journal of Neuroradiology, vol. 31, no. 6, pp. 1068-1075, 2010.

[119] R. L. Sacco, J. H. Ellenberg, J. P. Mohr et al., "Infarcts of undetermined cause: the NINCDS Stroke Data Bank," Annals of Neurology, vol. 25, no. 4, pp. 382-390, 1989. 


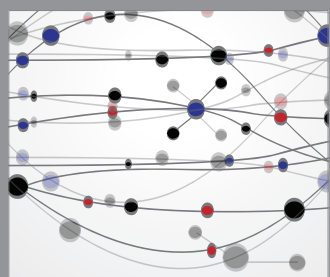

The Scientific World Journal
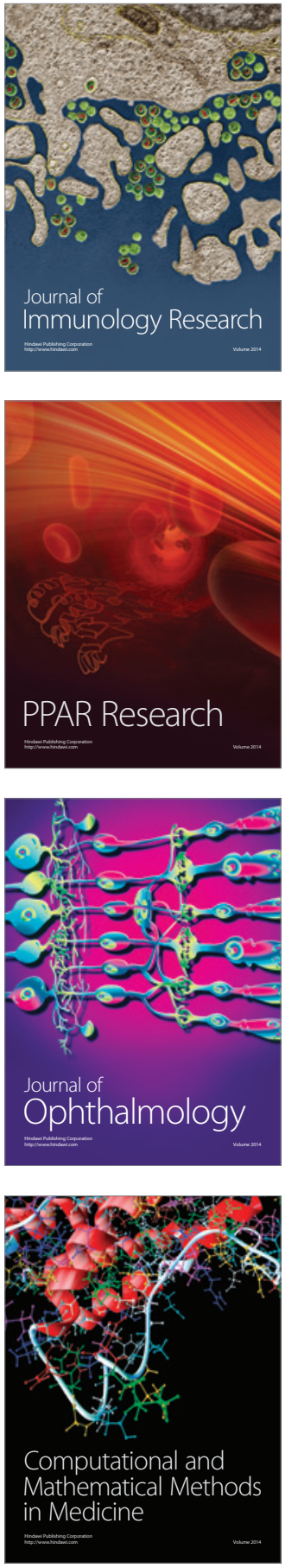

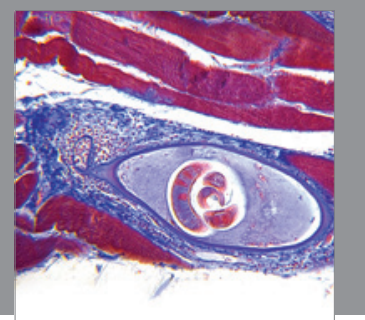

Gastroenterology

Research and Practice
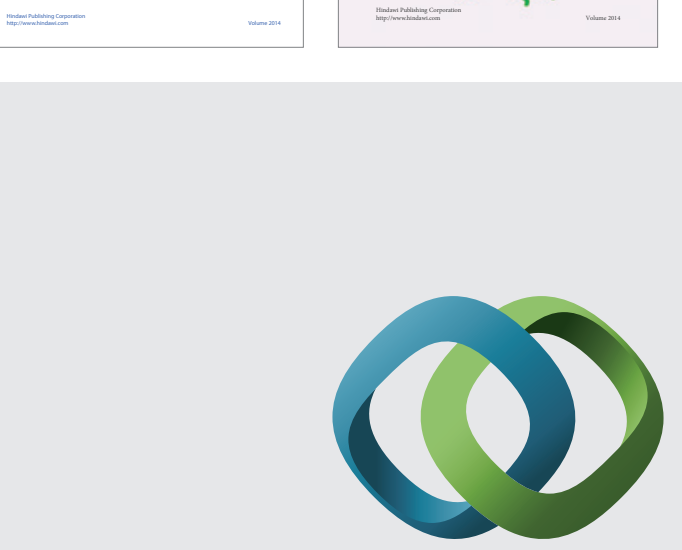

\section{Hindawi}

Submit your manuscripts at

http://www.hindawi.com
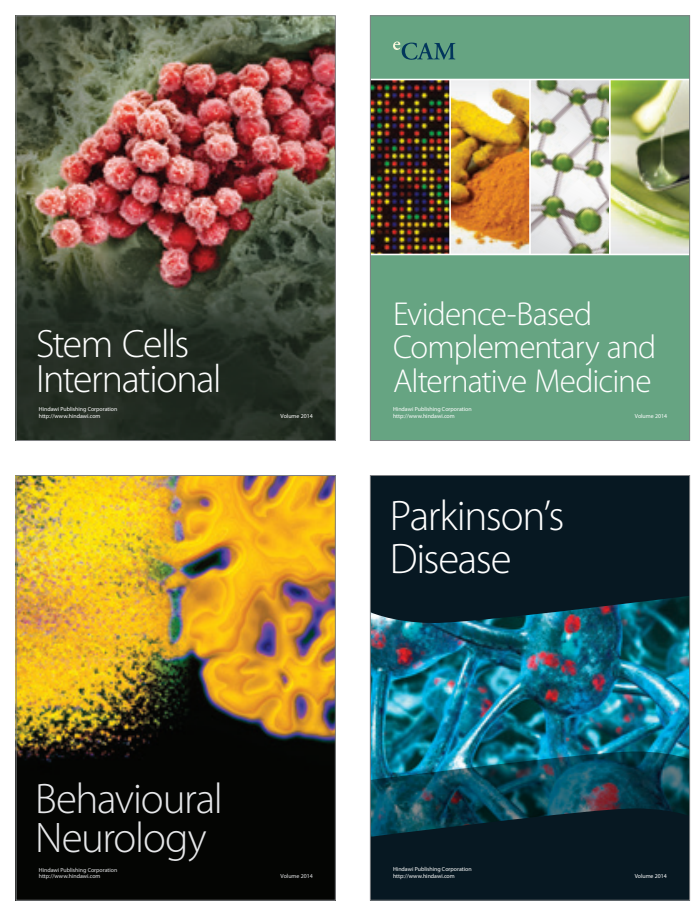

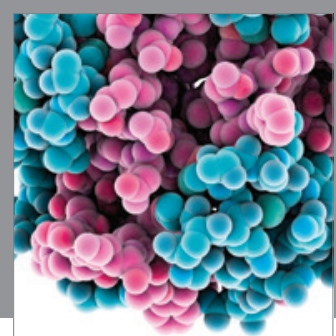

Journal of
Diabetes Research

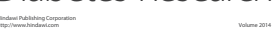

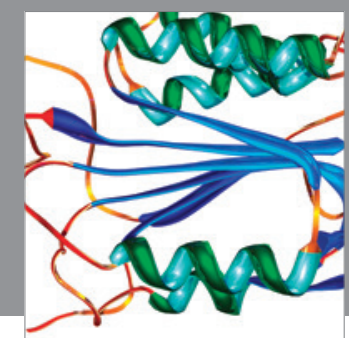

Disease Markers
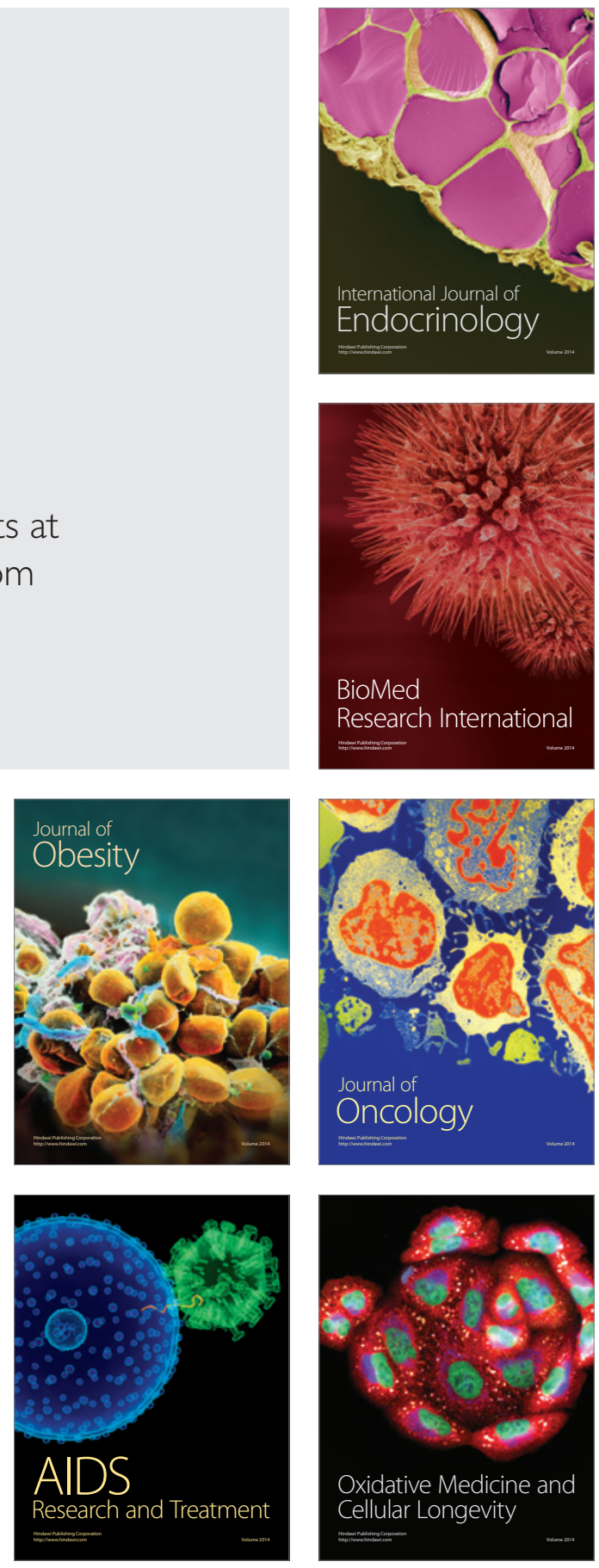